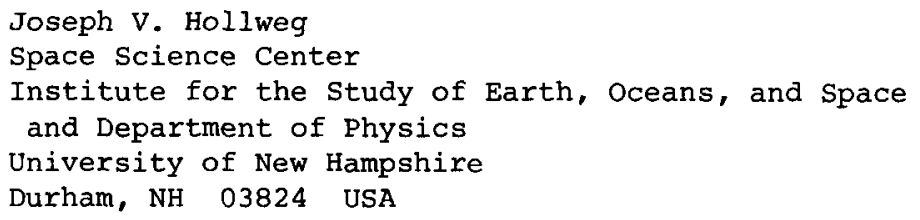

\title{
Introduction
}

After four decades of study, the mechanisms for heating the corona are not understood. However, the development of the field is vigorous. A variety of new ideas have been proposed, and these new ideas have generated lively debate. The goal of this short review is to present a broad overview of the ideas currently under consideration.

It is now accepted that the solar magnetic field is the key ingredient. It is not known whether waves are important or not, but it is agreed that the magnetic (and possibly associated kinetic) energy must eventually appear in thin structures so that the (generally weak) dissipative processes can heat the plasma. This poses a problem of how the heat gets distributed throughout the corona.

-2 The coronal heating requirements range from about $3 \times 10^{5}$ erg loops. From observations of the Doppler widths of spectral lines we have some knowledge of the "turbulent" kinetic energy in the corona (rms line-of-sight turbulent velocities are about $20-40 \mathrm{~km} \mathrm{~s}^{-1}$ ). If this kinetic energy were associated with sound waves, the wave energy flux density would be much too small. This is one reason for invoking the magnetic field. The other reason is from inspection of the remarkable $x$-ray images of the corona, which clearly show loops of plasma confined by the field. However, the corona outside of the loops also requires substantial energy. It is probably a mistake to isolate the coronal loop heating problem as many workers have tended to do. 
Observational constraints are very limited. There is general agreement ${ }^{1}$ that the heating is positively correlated with the magnetic field strength, $B$. And Parker ${ }^{2}$ has emphasized that the surface brightness of the $x$-ray loops is roughly independent of loop size, implying an inverse correlation of heating with loop length, L.

\section{Stressed Magnetic Fields}

There are difficulties with wave theories. One is that the waves must damp efficiently in the corona, e-folding in a few periods. This is a difficult (but not impossible) demand. On short loops, the transit time for an Alfven wave can be less than $10 \mathrm{~s}$. Unless there is substantial wave power at such short periods, it makes more sense to think of the field lines as simply being slowly displaced by the footpoint motions. If one footpoint moves with velocity $V_{h}$, the magnetic energy along the loop grows as $d(M E) / d t=h_{B}^{\prime} 2 v_{h}^{2} t / 4 \pi L$ ergs $\mathrm{cm}^{-2} s^{-1}$. The inverse dependence on $L$ is qualitatively desired. Parker has argued that random motions will inevitably produce coronal current sheets which lead to reconnection. He further argues that a steady-state will be achieved when $t=\overline{1}$ (flux tube diameter)/(velocity of reconnection). Thus $d(M E) / d t-\overline{1}\left(B^{2} / 4 \pi\right)$ $\left(v_{h}^{2} / v_{r e c}\right)(d / L)$. If we require $d(M E) / d t=-10^{7}$ erg $\mathrm{cm}^{-2} \mathrm{~s}^{-1}$, and take dy $=0.1, B=50 G$, and $v_{h}=0.4 \mathrm{~km} \mathrm{~s}^{-1}$, we find a steady-state after $90 \mathrm{hrs}$, when $\mathrm{v}_{\mathrm{h}} \mathrm{t}=0.2 \mathrm{r}_{\mathrm{h}}$. We also require $\left(\mathrm{v}_{\mathrm{rec}} / \mathrm{v}_{\mathrm{Alfven}}\right)=$ $10^{-5}$. Observationally, it is not known if these numbers alfe reasonable.

Parker's ideas have stirred up a controversy. He, B.C. Low, and others have grgued that current ${ }_{5}$ sheets are inevitable. A. van Ballegooijen ${ }^{4}$ and $\mathrm{s}_{5}$ Antiochos ${ }^{5}$ have argued that current sheets are not inevitable ${ }^{4}$, but ${ }^{5}$ are associated with surfaces of discontinuous magnetic connectivity or magnetic null points.

Van Ballegooijen ${ }^{6}$ has produced a statistical cascade model in which random walks at the footpoints produce a power spectrum for the current density which increases exponentially with time at large wave numbers. Eventually, joule dissipation at high wavenumbers gives a steady-state. He then obtains $\mathrm{d}(\mathrm{ME}) / \mathrm{dt} \approx 10^{5} \mathrm{erg} \mathrm{cm}^{-2} \mathrm{~s}^{-1}$ which is too small by two orders of magnitude.

Sturrock and Uchida ${ }^{7}$ considered twists produced on magnetic flux tubes by random walks of the footpoints; observational evidence for or against twists is needed. They obtain $d(M E) / d t=(B B * / 4 \pi) v_{h}^{2}$ $\tau / L$, where $B^{*}(\sim 1500 \mathrm{G})$ is the photospheric field strength. $\mathrm{h}$ If $\mathrm{B}^{\mathrm{CO}}={ }^{5} \mathrm{FG}_{\mathrm{G}} \mathrm{L}=10^{5} \mathrm{~km}_{6}^{2} \mathrm{v}_{\mathrm{h}}=1-\mathrm{km} \mathrm{s}_{1}^{-1}$ and $\tau_{\text {corr }}=300 \mathrm{~s}$, then $\mathrm{d}(\mathrm{ME}) / \mathrm{dt}=1.8 \times 10^{6} \mathrm{erg} \mathrm{cm}^{-2} \mathrm{~s}^{-1}$. This $\mathrm{Corr}_{\mathrm{s}}$. Toser to the required value of $10^{7}$, but still too small. A peculiar feature of this model is that there is no need to specify the dissipation mechanism.

Heyvaerts and Priest ${ }^{8}$ have produced a sophisticated model in which magnetic stresses repeatedly release energy subject to the 
conservation of magnetic helicity, leading to a constant- $\alpha$ force-free state after each relaxation. In a poster at this meeting, P.K. Browning applied these ideas to a cylindrical loop of radius $\mathrm{R}$ being twisted at a velogity $v$. She obtained a steady-state heating $d(M E) / d t=(8 / 75)\left(B^{2} v / 4 \pi\right)(R / L) \quad\left(\tau_{r e l} / \tau v^{\prime}\right)$ where $\tau_{r e l}$ is a relaxation time and $\tau_{\mathrm{v}}$ is a time scale for the twists; generally $\tau_{\text {rel }} / \tau_{\mathrm{y}}<1$. This expression is basically a measure of the Poynting lux into a loop with $\mathrm{B}_{\theta} / \mathrm{B} \approx \tau \mathrm{fe}_{1} / \tau \mathrm{y}^{\circ}$ If $\mathrm{B}_{6}=50 \mathrm{G}, \mathrm{v}_{2}=3 \rho \mathrm{km} \mathrm{s} \mathrm{s}^{-1}, \mathrm{R} / \mathrm{L}=0.1$, and $\tau$ rel $/ \tau=1$, we obtain $6.4 \times 10^{6}$ erg $\mathrm{cm}^{-2} \mathrm{~s}^{-1}$, which is close to the
observed values.

These models have their successes and failures. Except for the Browning calculation, the heating rates tend to be rather low. It is not clear what role they play in the chromosphere. It is generally agreed that these models fail on open magnetic field lines (e.g. coronal holes) where the stresses propagate away as Alfven waves.

These models do not include dynamics, although reconnegtion must certainly bring dynamics into play ${ }^{9}$. Numerical simulations ${ }^{9}$ will probably help in this regard. We should alsp mention a recent simulation by Mikic, Schnack, and van Hoven which showed the rms current density increasing exponentially with time, in qualitative agreement with van Ballegooijen's model. However, the simulation did not critically test other aspects of the model.

A strong plus for these models is evidence that some heating occurs impulsively, as might be expected if the reconnection leads to "microflares". Porter and Moore ${ }^{11}$ observed microflares in CIV occuring in low-lying $4(2-4000 \mathrm{~km}$ high) loops. They estimate that at any time there are $10^{4}$ events of $10_{5}^{26}$ erg on the sun. If each lasts $30 \mathrm{sec}$ the global average is $5 \times 10^{5} \mathrm{erg} \mathrm{cm} \mathrm{cm}^{-2} \mathrm{~s}^{-1}$. It is not yet clear if this number would approach $10^{7}$ if only the active regions were considered. It is also not known how the energy gets to higher heights; perhaps the impulses launch waves. (We also caution the reader that these events are not guaranteed to be due to reconnection; short-lived dynamics events could also be waves or shocks.)

\section{Wave Theories}

Wave theories may seem old-fashioned, but there are still reasons for considering them. The most compelling reason is that Alfven waves have been observed in the solar wind. There are other reasons as well. It is generally agreed that waves are needed to heat the open coronal field lines. We know that there are "turbulent" motions in the corona; if the observed motions are assumed to propagate at the Alfven speed, then the energy fluxes are adequate to heat the corona. There are some hints of wave-excited resonances on active region loops ${ }^{12}$. There are other dissipation mechanisms besides electrical resistivity and reconnection. And 
because the waves propagate through the photospheric flux tubes and chromosphere, they can be expected to heat those regions as well.

One wave damping mechanism of çurrent interest is resonance absorption either of surface waves ${ }^{13,14}$ or of waves propagating towgrd a smooth boundary. For a coronal surface wave, the damping is $\gamma / \omega=\pi k a\left(\rho_{2}-\rho_{1}\right) / 8\left(\rho_{2}+\rho_{1}\right)$, where 'a' is the surface thickness. For typical numbers, the wave can e-fold in a few periods as required. It has also been pointed out ${ }^{14}$ that resonance absorption can give a surface brightness independent of loop length. Resonance absorption dumps the surface wave energy into a thin layer, but that in turn could lead to turbulence which could actually distribute the energy over a substantial fraction of a loop's diameter ${ }^{14}$.

Turbulence is also a possible damping mechanism for waves. This is poorly understood theoretically, but it does seem to be happening in the solar wind, where we see waves but with a Kolmogorov turbulent $\left(\mathrm{k}^{-5 / 3}\right)$ power spectrum. The ghserved wave damping corresponds to the turbulent heating rate $\rho<\delta \mathrm{v}^{2}>3 \% / \ell$, where leorr is the correlation length. It turns out Ehâ this heaEing rate "works" almost everywhere in the solar atmosphere, but it fails in coronal holes where the turbulent heating produces a large proton temperature peak near $3 r_{0}$, if it is assumed that the energy eventually heats the protons.

\section{References}

1. Golub, L., Maxson, C., Rosner, R., Serio, S., and Viana, G.S.: 1980, Ap.J., 238, pp. 343-348.

2. Parker, E.N.: 1986, in Coronal and Prominence Plasmas, NASA-CP2442 , pp. 9-17.

3. Parker, E.N.: 1983, Ap.J., 264, pp. 642-647.

4. van Ballegooijen, A.A.: 1985, Ap.J., 298, pp. 421-430.

5. Antiochos, S.K.: 1988, Bull. Amer.Astron. Soc., 20, p. 681.

6. van Ballegooijen, A.A.: 1986, Ap. J., 311, pp. 1001-1014.

7. Sturrock, P.A., and Uchida, Y.: 1981, Ap.J., 246, pp. 331-336.

8. Heyvaerts, J., and Priest, E.R.: 1984, Astron. Astrophys, 137, p. 63 .

9. Dahlburg, R.B., Dahlburg, J.P., and Mariska, J.T.: 1988, Astron. Astrophys., in press.

10. Mikic, Z., Schnack, D.D., and van Hoven, G.: 1988, Ap.J., submitted.

11. Porter, J.G., and Moore, R.L.: 1987, NASA-Marshall preprint 87-146.

12. Koutchmy, S., Zugzda, Y.D., and Locans, V.: 1983, Astron. Astrophys., 120, p. 185.

13. Davila, J.M.: 1987, Ap.J., 317, pp 514-521.

14. Hollweg, J.V., and Yang, G.: 1988, J. Geophys. Res., 93, pp. 5423-5436.

15. Hollweg, J.V., and Johnson, W.: 1988, J. Geophys. Res., in press. 